\title{
Aging Behaviour of Polypropylene under Various Voltage Stresses
}

\author{
Weijia Zhao ${ }^{1}$, Wah Hoon Siew ${ }^{1}$, Martin J Given ${ }^{1}$, Qingmin Li $^{2}$, Jinliang $\mathrm{He}^{3}$ and Edward Corr ${ }^{1}$ \\ 1. Department of Electronic \& Electrical Engineering, University of Strathclyde \\ 2. Beijing Key Laboratory of High Voltage \& EMC, North China Electric Power University \\ 3. State Key Lab of Power Systems, Department of Electrical Engineering, Tsinghua University \\ weijia.zhao@strath.ac.uk,wh.siew@strath.ac.uk,m.given@strath.ac.uk, lqmeee@ncepu.edu.cn, hejl@tsinghua.edu.cn and \\ edward.corr@strath.ac.uk
}

\begin{abstract}
Practical HVDC systems have superimposed AC or transients and the synergistic effect of these factors on polymer aging would be of interest. Although it is clear that partial discharges under AC stress will gradually degrade the insulation behavior of a polymeric material, there are not many publications detailing the effect of superimposed AC voltages on polymer performance in a HVDC power system. Assuming polypropylene (PP) is suitable for use as electrical insulation, this paper considers the behavior of $P P$ under various voltage ratios and temperatures. Factors which cause the degradation of PP will be summarized and explained. To simulate the working condition, electro-thermal aging equipment will be used. Fourier transform infrared spectroscopy - attenuated total reflection (FTIR-ATR) measurement and dielectric spectroscopy measurement will be carried out before and after aging.
\end{abstract}

\section{INTRODUCTION}

Initially, polymeric insulation [1] and insulation based on rubber composites were mainly used in low-voltage power cables. The trend to date has been to develop the technology further for use in medium and high voltage AC power cables [2].

The move towards isolated renewable generation has brought the requirement to transmit power over longer distances to centers of demand. High voltage direct current (HVDC) transmission enables a lower loss transmission system than conventional high voltage alternating current (HVAC) transmission. The most promising renewable generation technique is that of wind power and in particular offshore wind where HVDC transmission is likely to be utilized.

In a HVDC transmission system connected to an offshore windfarm, each side of HVDC link has a converter station. The offshore substation contains the AC/DC converter and the onshore substation has the DC/AC converter. The converter station is the key component in HVDC system used to switch from $\mathrm{AC}$ to $\mathrm{DC}$ voltage and vice versa. Classic HVDC systems are based on Line Commutated Converters (LCC), while modern HVDC systems use voltage source converters (VSC). However, both techniques superimpose AC signals on the DC output voltage [3].

The switching of the power electronics leads to a DC output voltage with superimposed transients. The switching frequency can be in the $\mathrm{kHz}$ range [4]. The $\mathrm{AC}$ components contain various voltage amplitudes and frequencies. This may be a particular challenge for subsea cable insulations. As such it is useful to investigate the performance of polymer affected by these combined voltages.

Researchers have performed investigations on the behavior of oil-pressboard under individual DC, AC, impulse voltages or combined voltages. Under AC and DC combined voltages, the partial discharge feature of voids in oil-pressboard was summarized in [5-6]. The results demonstrated that the partial discharge (PD) inception voltage increased and the PD charge decreased along with an increase in the DC voltage relative to the total voltage stress of AC and DC combined voltages.

Previous work has also been conducted on the electrical breakdown characteristics of oil-paper under AC and DC combined stresses. Wang et al. [7] reported that the value of DC breakdown strength of the oil-paper is higher than the value of $\mathrm{AC}$ breakdown strength. The value of $\mathrm{AC}$ and $\mathrm{DC}$ combined breakdown strength was between the DC value and the AC value. Wang et al. [8] also investigated the breakdown characteristic of the oil-paper aged under AC and DC combined stresses. The work kept AC voltage, DC voltage and AC superimposed with DC voltage constant. The results showed that as the DC component increased for the combined voltage stresses, breakdown strength of oil-paper insulation increased.

The investigation on the behavior of HDPE aged under DC and $\mathrm{AC}$ combined stress with various frequencies was reported in [9]. The results demonstrated that the DC voltage could encourage cross-linking and AC voltage could encourage degradation. The degradation process developed further as AC frequency increased.

However, there are not many publications on how polymer properties change with different DC\%. This is the aim of this paper. This paper will investigate how dielectric properties of polymers change after AC and DC stress aging. In this paper, polypropylene (PP) is assumed suitable for use as electrical insulation. To model the real condition on cable insulation, thermal-electrical aging stresses were carried out on PP samples. The frequency was set at $1 \mathrm{kHz}$ with various DC to total voltage ratios. In this paper, the DC\% was $37.5 \%$, $43.75 \%, 50 \%, 56.25 \%$ and $62.5 \%$. FTIR-ATR measurement and dielectric spectroscopy measurement were carried out before and after aging. 


\section{EXPERIMENT SET-UP}

The PP film, $60 \times 60 \mathrm{~mm}$ in geometry and $200 \mu \mathrm{m}$ in thickness, was sandwiched between sphere-plane electrodes, as shown in Fig.1.

Both electrodes were made of stainless steel. The sphere electrode was $20 \mathrm{~mm}$ in diameter, which was connected to the high voltage power supply. The plane electrode was connected to ground.

To model the real condition of subsea cable insulation, thermal stress was applied to the samples with electrical stress simultaneously. To control the surrounding temperature, the electrodes were placed in an oven. Therefore, the thermalelectrical aging system is established.

To see the long-term degradation of PP in a short time, it is essential to carry out accelerated aging. As the operating temperature of a $\mathrm{HVDC}$ cable is $70^{\circ} \mathrm{C}$ the temperature should be below the melting temperature of PP. As the melting temperature $T_{m}$ of $\mathrm{PP}$ is around $160^{\circ} \mathrm{C}$. The aging temperature was set to $110^{\circ} \mathrm{C}$. Each PP sample was aged in the thermalelectrical box for 1 hour.

The waveform of AC and DC combined voltage is detailed in Fig.2.

The combined voltage was generated by a signal generator and input to a power amplifier with a gain of 5000 .

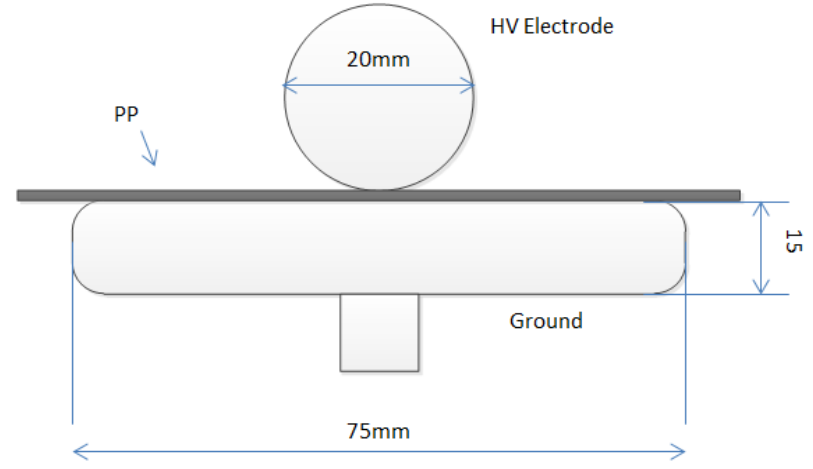

Fig. 1. Electrode of thermal-electrical aging

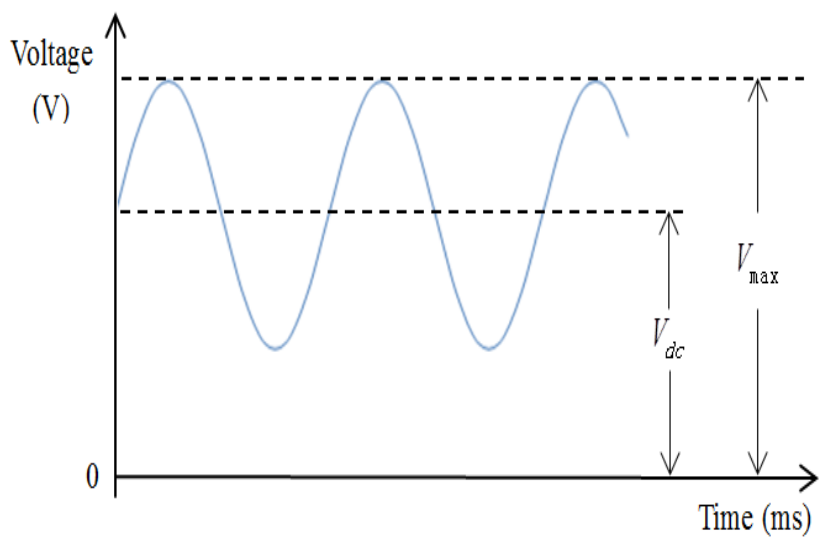

Fig. 2. AC\&DC combined voltage waveform
To define the combined voltage stress, the DC percentage is detailed in equation 1.

$$
D C \%=\frac{V_{d c}}{V_{\max }} \times 100 \%
$$

In equation $1, \mathrm{~V}_{\mathrm{dc}}$ is the mean value of $\mathrm{DC}$ voltage and $\mathrm{V}_{\max }$ is the maximum value of the $\mathrm{AC}$ and $\mathrm{DC}$ combined voltage. In this paper, $V_{\max }$ is fixed at $5 \mathrm{kV}$.

The FTIR-ATR measurement was used to measure the chemical properties of the sample. Dielectric spectroscopy was used to measure the dielectric properties (real permittivity, imaginary permittivity and dissipation factor) by applying a IV AC voltage onto the sample via parallel electrodes.

\section{RESUlTS AND DISCUSSIONS}

The results of FTIR-ATR measurement and dielectric spectroscopy results are presented in this section.

\section{A. FTIR-ATR Results}

The FTIR-ATR results of the reference and aged PP are shown in Fig.3-5.

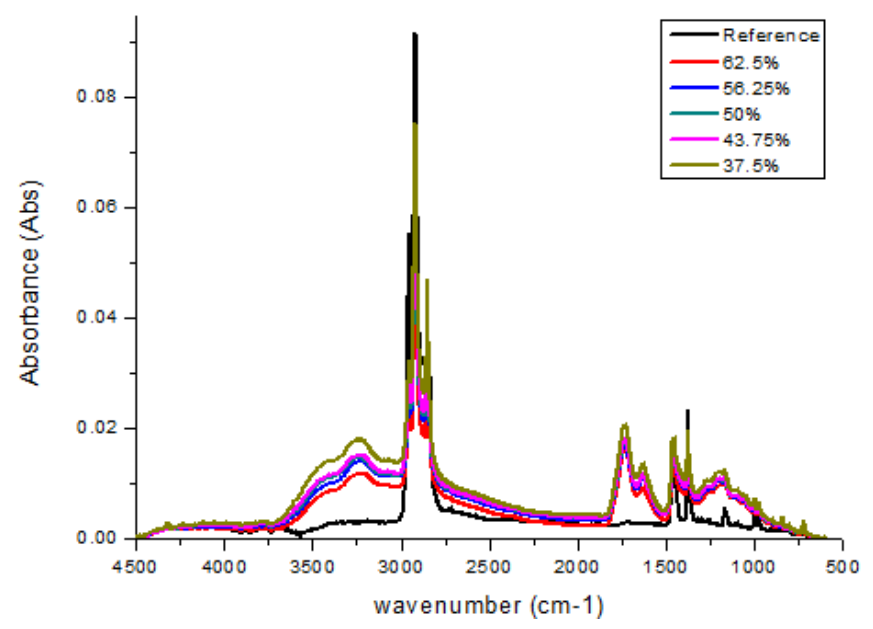

Fig. 3. FTIR-ATR results for PP

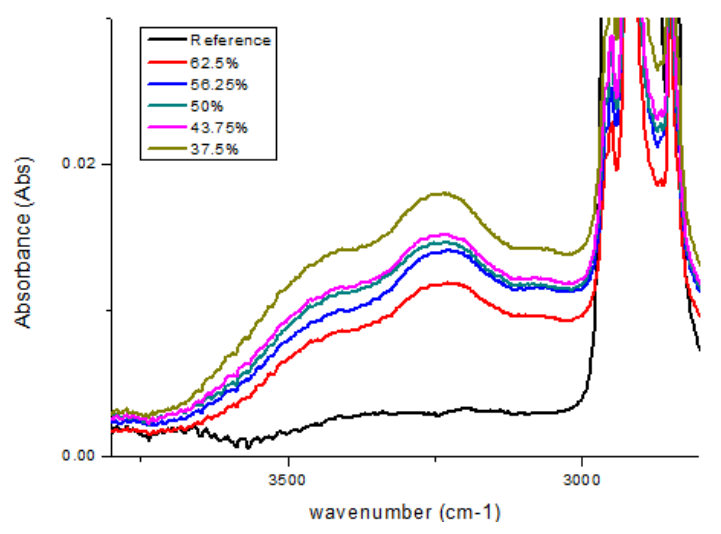

Fig. 4. FTIR-ATR results for PP detailed view of OH-group 


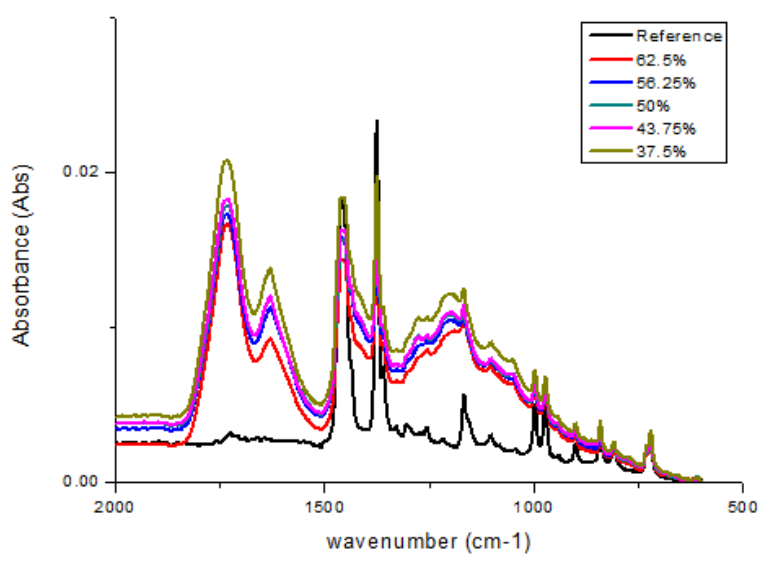

Fig. 5. FTIR-ATR results for PP detailed view of carbonyl group and CO group

In the reference sample, original peaks are shown in Table I. In the wavenumber range of $3000-2800 \mathrm{~cm}^{-1}$ the bond vibration is mainly of $\mathrm{C}-\mathrm{H}$ stretching. In the wavenumber range of $1500-1350 \mathrm{~cm}^{-1}$ the bond vibration mainly describes $\mathrm{C}-\mathrm{H}$ bending.

Compared with the reference sample, new peaks appear in the $3600 \sim 3100 \mathrm{~cm}^{-1}$ range and $1800 \sim 1600 \mathrm{~cm}^{-1}$ range in thermal-electrical aged samples. Between them, the former demonstrates the hydroxyl group $(\mathrm{O}-\mathrm{H})$, the latter demonstrates the carbonyl groups $(\mathrm{C}=\mathrm{O})$. The detailed views of these wavenumber ranges are shown in Fig. 4 and Fig.5.

The intensity of absorbance corresponds to the amount of molecular groups. Across the wavenumber ranges of $3600 \sim 3100 \mathrm{~cm}^{-1}$ and $1800 \sim 1600 \mathrm{~cm}^{-1}$ it is obvious that the intensities of the aged samples are much higher than the reference sample. When the DC\% was decreased the intensity of the new peak increased. This means that more hydroxyl group $(\mathrm{O}-\mathrm{H})$ and carbonyl groups $(\mathrm{C}=\mathrm{O})$ were created under combined stresses.

The new kinds of molecules are probably due to the decomposition of the reference PP. When PP was aged under thermal-electrical stress, chain scission worked on the long chain of PP. The oxidation process may act at the new terminals, oxygen combined with $\mathrm{H}$ and $\mathrm{C}$ at the terminals and $\mathrm{O}-\mathrm{H}$ and $\mathrm{C}=\mathrm{O}$ groups were created. The increased number of new molecules leads to further oxidation and more breaks in the long chains. This leads to increased degradation in the material.

TABLE I

Bond Vibration AND WaVEnumber [10]

\begin{tabular}{|c|c|}
\hline Wavenumber $\left(\mathrm{cm}^{-1}\right)$ & Bond vibration \\
\hline 2950 & $\mathrm{CH}_{3}$ asymmetric stretching \\
\hline 2918 & $\mathrm{CH}_{2}$ asymmetric stretching \\
\hline 2873 & $\mathrm{CH}_{3}$ symmetrical stretching \\
\hline 2848 & $\mathrm{CH}_{2}$ symmetrical stretching \\
\hline 1459 & $\mathrm{CH}_{2}$ bending \\
\hline 1375 & $\mathrm{CH}_{3}$ symmetrical deformation \\
\hline 1166 & $\mathrm{CH}_{3}$ wagging \\
\hline 972 & $\mathrm{CH}_{3}$ rocking \\
\hline
\end{tabular}

The presence of polar molecules may result in a change of dielectric properties.

\section{B. Dielectric Spectroscopy Results}

The dielectric properties of PP are presented in Fig. 6-8. The properties presented are real permittivity (Fig. 6), imaginary permittivity (Fig. 7) and dissipation factor (Fig. 8).

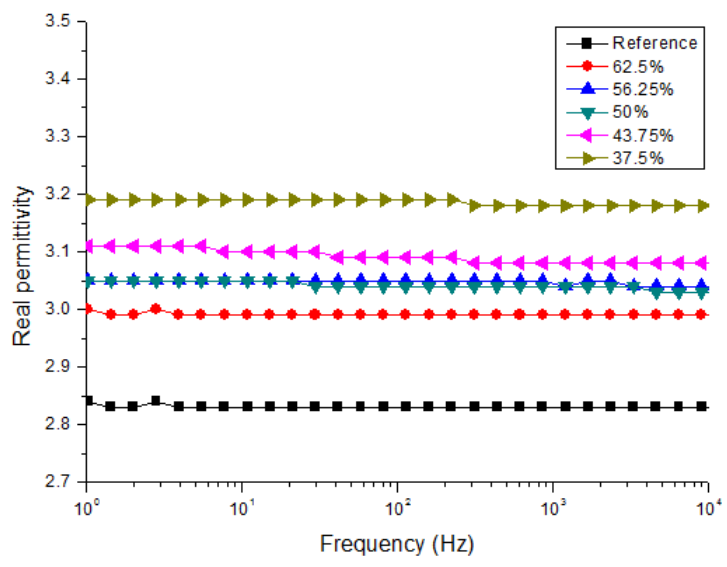

Fig. 6. Real permittivity of aged $\mathrm{PP}$ under $1 \mathrm{kHz}$

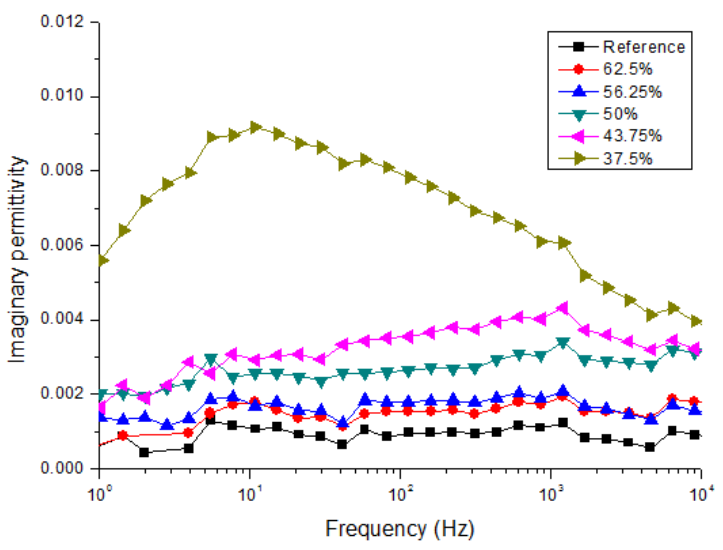

Fig. 7. Imaginary permittivity of aged $\mathrm{PP}$ under $1 \mathrm{kHz}$

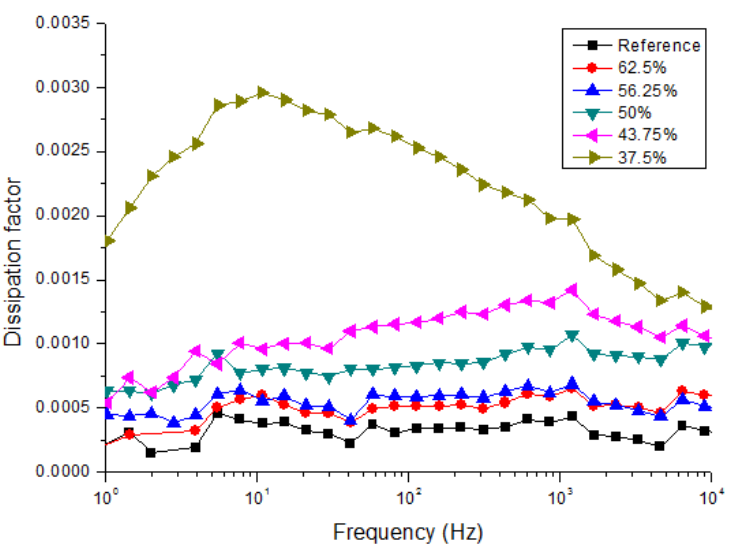

Fig. 8. Dissipation factor of aged PP under $1 \mathrm{kHz}$ 
Real permittivity $\left(\varepsilon^{\prime}\right)$ is the dielectric constant of the material. In the $10^{0}$ to $10^{4} \mathrm{~Hz}$ range, $\varepsilon^{\prime}$ is nearly a constant for each sample. For each sample, $\varepsilon^{\prime}$ slightly increases as the frequency decreases. The value of $\varepsilon^{\prime}$ for the reference and aged samples vary between 2.8 to 3.2. This result agrees with the variation expected for the dielectric constant with frequency. The orientation polarization of the polar molecule is influenced by frequency and to a certain extent, the dielectric constant decreases as frequency increases [3].

The $\varepsilon^{\prime}$ of the reference is approximately 2.85 . The dielectric constants of all aged samples were higher than the reference. When PP was aged under DC $62.5 \%$, the value of $\varepsilon^{\prime}$ increases to 3.0. As DC\% was further decreased to $56.25 \%, \varepsilon^{\prime}$ increased to around 3.1 and remained stable when DC\% decreased to $50 \%$. As DC\% decreases further the value of $\varepsilon^{\prime}$ increases. The value of $\varepsilon^{\prime}$ reaches the highest value when DC\% was 37.5.

When AC stress was applied to the PP, partial discharge was encouraged as the AC voltage was increased. The partial discharge activity experienced by the polymer leads to further degradation of the material. This degradation was the main process which acted to age the polymer. Along with the degradation mechanism, the chain scissions will act so that long chains breaks into small chains. Oxidation normally takes place at the terminals and polar molecules are created, such as $\mathrm{O}-\mathrm{H}, \mathrm{C}=\mathrm{O}$, which will increase the orientation polarization and increase the dielectric constant further.

The aging mechanism is strongly dependent on the DC\%. According to Fig.6, thermal-electrical stresses encourage degradation. Therefore, $\varepsilon^{\prime}$ of the aged sample is higher than the reference sample. As the DC\% is reduced more polar molecules are created leading to an increase of $\varepsilon^{\prime}$. The results are consistent with the FTIR-ATR results.

In the $10^{0}$ to $10^{4} \mathrm{~Hz}$ range, dissipation factor $(\tan \delta)$ of the reference sample and most of the aged samples remain constant except for the sample aged at $37.5 \%$ DC percentage, as shown in Fig.8. The $\tan \delta$ values of the aged samples were higher than the reference sample. In addition, as the DC\% decreases, the $\tan \delta$ value increases.

The dielectric results are in accordance with the FTIR-ATR results.

\section{CONCLUSIONS}

This paper has investigated the change in chemical and dielectric properties of PP at $1 \mathrm{kHz}$ with various DC\% when subjected to thermal-electrical aging. Chemical and dielectric properties were assessed using FTIR-ATR and dielectric spectroscopy respectively.

The results from FTIR-ATR measurements shows that when the DC\% was decreased the intensity of the hydroxyl group $(\mathrm{O}-\mathrm{H})$ and the carbonyl groups $(\mathrm{C}=\mathrm{O})$ increased.

The results from dielectric spectroscopy tests show that the dielectric constant and dissipation factor reduced as the DC\% was reduced. This is because as the DC\% was increased, less $\mathrm{O}-\mathrm{H}$ groups and $\mathrm{C}=\mathrm{O}$ groups were created. As the number of polar molecular groups decrease, the dielectric constant and dissipation factor reduced.

\section{ACKNOWLEDGMENT}

The facilities provided by Tsinghua University, China and North China Electric Power University, China are gratefully acknowledged.

\section{REFERENCES}

[1] W. Zhao et al., "The Electrical Performance of Thermoplastic Polymers When Used As Insulation in Cables," in International Universities' Power Engineering Conference, Dublin, 2013, pp. 1-4.

[2] IEEE Committee Report, "History and Design Parameters of Low- and Intermediate-Pressure Oil-Filled Cable in the Western Hemisphere Operating at $60 \mathrm{kV}$ and Above," 1969, vol. PAS-88, Issue: 5 , Part: Part-I, pp. 541-557.

[3] F. Mauseth et al., "Water Tree Growth of Wet XLPE Cables Stressed with DC and High Frequency AC Voltage Superimposed," in Electrical Insulation, 2012, pp. 266-269.

[4] H. H. Sæternes et al., "Water Treeing in XLPE Insulation at a Combined DC and High Frequency AC Stress," in Electrical Insulation Conference, 2013, pp. 494-498.

[5] B. Qi et al., "Influences of Ratios of AC and DC Voltages on Partial Discharge Characteristics of Gas Cavity in Oil-pressboard Insulation," in Proceedings of the CSEE, 2015, vol.35, no.1, pp. 247-254. In Chinese.

[6] B. Qi et al., "Discharge Characteristics of Internal Gas-gap Defect in Oil-paper Insulation Under Different Proportion of AC and DC Combined Voltages," in Proceedings of the CSEE, 2015, vol.35 no.12, pp. 3161-3169. In Chinese.

[7] Y. Wang et al., "Electrical Breakdown Properties of Oil-paper Insulation under AC-DC Combined Voltages," pp. 115-118.

[8] Y. Wang et al., "Failure Evaluation Model of Oil-Paper Insulation under AC-DC Combined Voltages," in International Conference on High Voltage Engineering and Application, Shanghai, China, 2012, pp. 396399.

[9] W. Zhao et al., "Thermoplastic Materials Aging under Various Stresses," in Electrical Insulation Conference, Montréal, Canada, 2016, pp. 615-618.

[10] R. Morent et al., "Comparison between XPS- and FTIR-analysis of plasma-treated polypropylene film surfaces," in Surface and Interface Analysis, 2008, Vol. 40, pp. 597-600. 